\title{
Effect of Primary Recrystallized Texture on the Behavior of Secondary Recrystallization in $3 \%$ Silicon Steel*
}

\author{
By Masatoshi Shinozaki**, Isao Matoba**, Takahiro Kan** \\ and Tomomichi Gotoh**
}

\begin{abstract}
Primary recrystallized textures of $3 \%$ silicon steel sheets cold-rolled to various directions and subsequently decarburized were analyzed by three dimensional crystalline orientation distribution function. Orientation, size and incubation period of secondary recrystallized grains were observed after final secondary recrystallization annealing. They were compared with the orientation distribution of primary recrystallized grains having a peak orientation near $\{110\}\langle 001\rangle$ and $\{111\}\langle 112\rangle$, the latter of which is a main component in the primary recrystallized texture. The behaviour of secondary recrystallization was discussed with special reference to the shapes of orientation distribution curves of primary recrystallized grains. The grain size, orientation distribution and other characters of secondary recrystallization are found to have a close correlation with the two primary recrystallization texture components, $\{110\}\langle 001\rangle$ and $\{111\}\langle 112\rangle$. This correlation was successfully explained in terms of some coincidence relations concentrated near $35^{\circ}$ about the $\langle 110\rangle$ axis.
\end{abstract}

(Received October 1, 1977)

\section{Introduction}

The texture formation mechanism in secondary recrystallization on silicon steel still remains unclarified to date, because conventional methods for representing primary recrystallized textures such as pole figures are not able to represent the exact textures.

Recently, the three dimensional crystalline orientation distribution function $(\mathrm{ODF})^{(\mathbf{1})(2)}$ was developed as an efficient method for quantitatively representing textures in terms of the calculated density of an arbitrary orientation. ODF has been mainly used to study the textures of low cabon steel sheets, but more recently it is also employed to examine the textures of $3 \%$ silicon steel sheets. For instance, Flowers and Heckler ${ }^{(3)}$ investigated a primary recrystallized texture of $3 \%$ silicon steel prepared by one- or two-stage cold rolling, and they calculated the volume percent of primary recrystallized grains with near $\{110\}\langle 001\rangle$ orientation. Kan et al. ${ }^{(4)}$ found that the orientation dis-

* Represented at the annual meeting of Japan Institute of Metals, April, 1976.

** Research Laboratories, Kawasaki Steel Corporation, 1, Kawasaki-cho, Chiba 280, Japan.

Trans. JIM tribution of secondary recrystallized grains are correlated with that of primary recrystallized grains having a near- $\{110\}\langle 001\rangle$ orientation in their study of two-stage cold rolling of $3 \%$ silicon steel. In the present study, the present authors intended to utilize the same method, but the cold rolling of the second stage was carried out in various directions with respect to the hot rolling direction. Shapes of orientation distribution curves of primary recrystallized grains with a near- $\{110\}\langle 001\rangle$ orientation and a near- $\{111\}\langle 112\rangle$ orientation, the latter of which is a main component in primary recrystallized textures, was noticed in relation to the orientation of secondary recrystallized grains, because the secondary grains are known to be certain primary recrystallized grains which grow at the expense of other primary grains. Some conclusions could be drawn on the texture formation mechanism of secondary recrystallization in $3 \%$ silicon steel.

\section{Experimental Procedure}

Compositions of the hot-rolled $3.0 \mathrm{~mm}$ thick specimens are given in Table 1 . They were annealed at $900^{\circ} \mathrm{C}$ for $3 \mathrm{~min}$, cold-rolled at the first stage to $0.75 \mathrm{~mm}$ thickness and then an-

1978 Vol. 19 
Table 1 Chemical composition of specimens (wt \%).

\begin{tabular}{ccccccc}
\hline $\mathrm{C}$ & $\mathrm{Si}$ & $\mathrm{Mn}$ & $\mathrm{P}$ & $\mathrm{S}$ & $\mathrm{Se}$ & $\mathrm{Sb}$ \\
\hline 0.032 & 3.02 & 0.06 & 0.01 & 0.005 & 0.02 & 0.03 \\
\hline
\end{tabular}

nealed at $925^{\circ} \mathrm{C}$ for $5 \mathrm{~min}$. The specimens were cut from the sheets in various directions of $0^{\circ}$ to $90^{\circ}$ with respect to the hot rolling direction and then subjected to the second stage of cold rolling to a final thickness of $0.30 \mathrm{~mm}$ in each direction. The cold-rolled sheets were decarburized at $820^{\circ} \mathrm{C}$ for $5 \mathrm{~min}$ in wet $\mathrm{H}_{2}$ atmosphere. The textures of the decarburized sheets were represented by (200) pole figures and ODF with regard to the surface and central layers. Since the large difference in primary recrystallized textures was not detected, in a preliminary examination, between the surface and the central layer, the textures were investigated only at the surface layer. The decarburized sheets were finally annealed at $860^{\circ} \mathrm{C}$ for $50 \mathrm{~h}$ in dry $\mathrm{H}_{2}$ atmosphere in order to complete the secondary recrystallization in the whole specimen. The orientation of secondary recrystallized grains was determined by using a special X-ray diffractometer developed by Abe $e t a l .^{(5)}$, and also the size of individual secondary recrystallized grains was measured. The incubation period of individual secondary recrystallized grains was measured in isothermal annealing at $890^{\circ} \mathrm{C}$. Finally, these behaviors of secondary recrystallization were compared with the orientation distribution curve of primary recrystallized grains.

\section{Experimental Results}

Secondary and primary recrystallized textures of the specimens cold-rolled in the directions of $0^{\circ}, 45^{\circ}$ and $90^{\circ}$ with respect to the hot rolling direction are shown in Fig. 1. Representation of the textures is based on the cold rolling direction of the second stage. Both of the secondary and primary recrystallized textures were symmetrical with respect to RD and TD in the case of cold rolling directions of $0^{\circ}$ and $90^{\circ}$. Orientations of the secondaries were distributed mostly in the vicinity of $\{110\}\langle 001\rangle$ orientation and the main components of the primaries were $\{111\}\langle u v w\rangle$ and $\{h k 0\}\langle 001\rangle$ orientations. But they were asymmetrical in the other case, showing an appreciable rotation from the above-mentioned textures about the

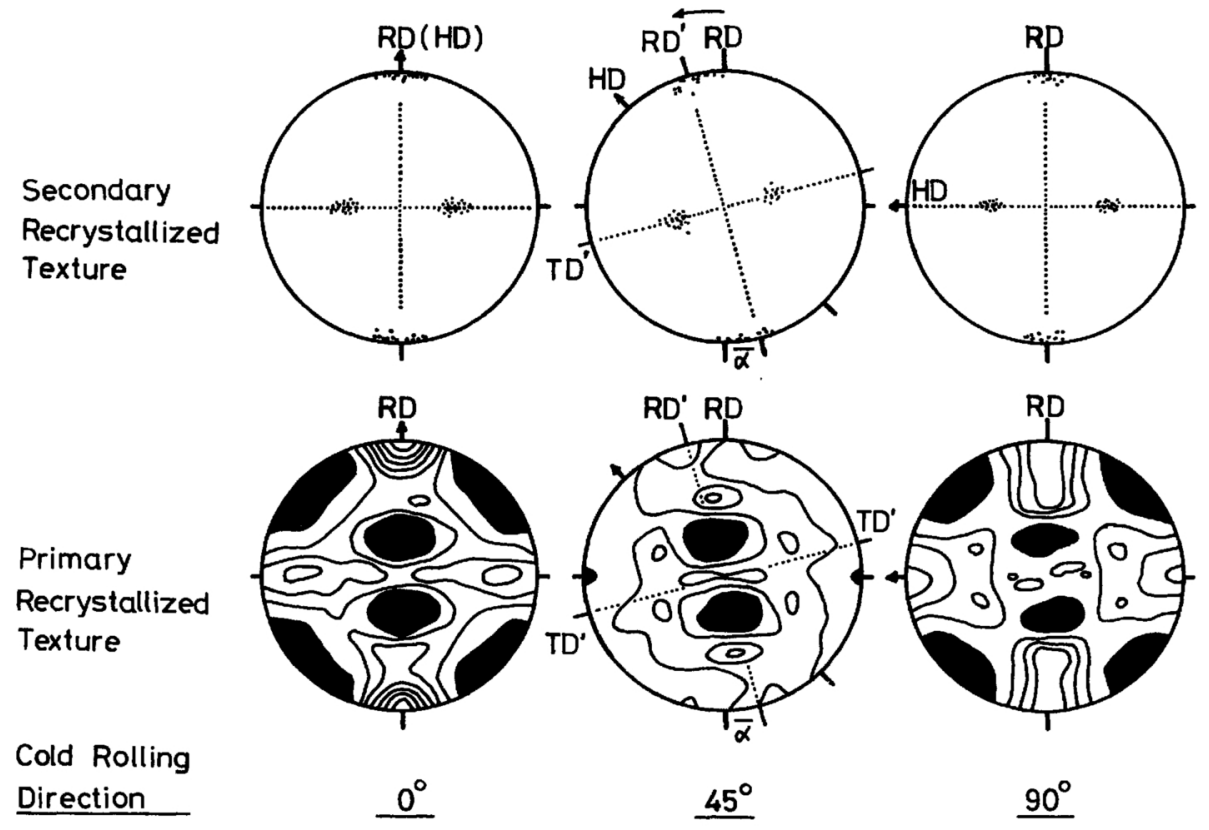

Fig. 1 (200) pole figures showing secondary and primary recrystallized textures. Arrow heads indicate the hot rolling direction (HD). 


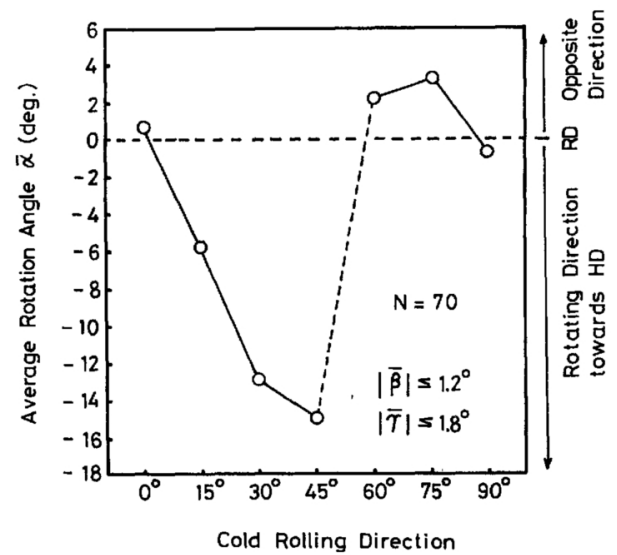

Fig. 2 Relation between average rotation angle $\bar{\alpha}$ about ND and the cold rolling direction.

sheet normal.

The following three angles were introduced for representing the deviations of individual secondaries from the ideal $\{110\}<001\rangle$ orientation

$\alpha:$ the deviation of the [001] axis from the rolling direction,

$\beta$ : the deviation of the [001] axis from the plane of the sheet,

$\gamma: \quad$ the angle between the (110) plane containing the [001] axis and the plane of the sheet.

The relation between the average angle of $\alpha$, $(\bar{\alpha})$, and the cold rolling direction is shown in Fig. 2. In the case of cold rolling directions of $0^{\circ}$ and $90^{\circ}$, the [001] axis in a large number of the secondaries were parallel to the cold rolling direction (RD). As the cold rolling direction deviated from the hot rolling direction (HD), the [001] axis of the secondaries deviated from $\mathrm{RD}$ to $\mathrm{HD}$, and $|\bar{\alpha}|$ attained a maximum value in the case of the cold rolling direction of $45^{\circ}$. On the contrary, in the cold rolling directions of $60^{\circ}$ and $75^{\circ}$, the [001] axis deviated slightly from $\mathrm{RD}$ to the opposite direction with respect to $\mathrm{HD}$. Thus, $\bar{\alpha}$ changed in response to the change of the cold rolling direction, but $\bar{\beta}$ and $\bar{\gamma}$ did not show any change and were nearly $0^{\circ}$ regardless of the cold rolling directions.

A special ODF program was developed in order to calculate primary recrystallized textures which had asymmetrical orientation

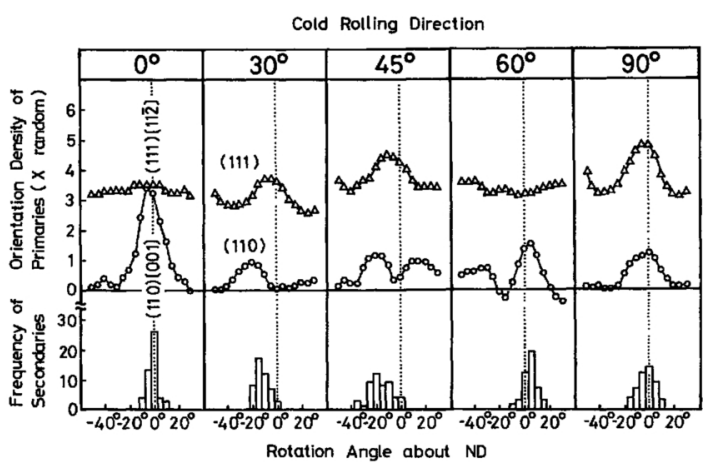

Fig. 3 Comparison between orientation distribution (frequency) of secondaries and orientation density of primaries about ND. Histograms: secondaries, circles: $\{110\}\langle u v w\rangle$ primaries, triangles: $\{111\}$ $\langle u v w\rangle$ primaries.

distributions with respect to RD. The orientation density of the primaries with three series of rotation from the $\{110\}\langle 001\rangle$ or $\{111\}\langle 112\rangle$ orientation about ND, TD and RD were calculated by the ODF. Main components in the primary recrystallized textures are wholly contained in the three orientation series. On the asymmetrical textures, two provisional directions were introduced for convenience of calculation as $\mathrm{TD}^{\prime}$ and $\mathrm{RD}^{\prime}$ which are defined as axes rotated from TD and $\mathrm{RD}$, respectively (see Fig. 1).

Figure 3 shows the orientation density of the primaries and the orientation distribution (frequency) of the secondaries as a function of orientation which is indicated by the rotation angle about ND. It should be noticed from Fig. 3 that the orientation distribution curve of the secondaries has the same shape as that of primaries with the $\{110\}$ component.

When the cold rolling direction was $0^{\circ}$ or $60^{\circ}$, the peak of orientation distribution of the secondaries and that of the primaries of the $\{110\}$ component were present on the same position. The orientation density of primaries of the $\{111\}$ component was strong and unchanged through the rotation about ND.

In the case of the $90^{\circ}$ cold rolling direction, the peak of the orientation distribution curve of the secondaries was in good agreement with that of the primaries of the $\{110\}$ and $\{111\}$ components.

When the cold rolling direction was $45^{\circ}$, two 


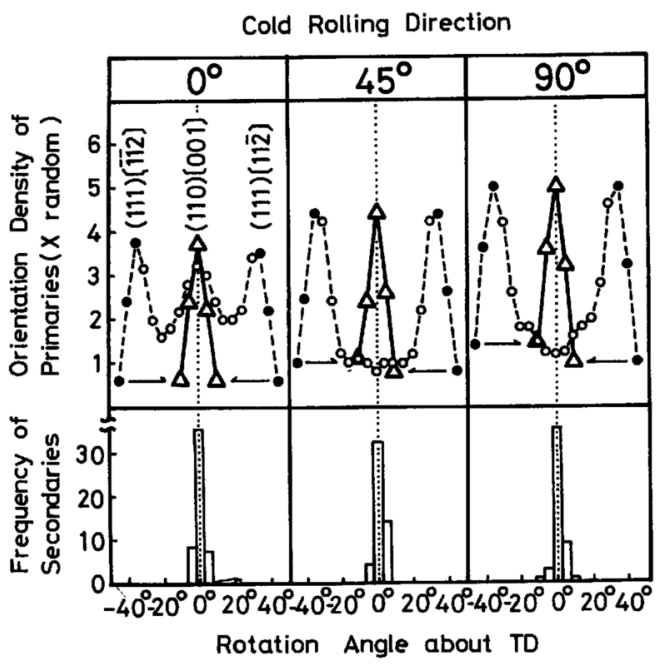

Fig. 4 Comparison between orientation distribution (frequency) of secondaries and orientation density of primaries about TD or TD'. Histograms: secondaries, circles: primaries having orientation $\langle 110\rangle / / \mathrm{TD}$ or $\mathrm{TD}^{\prime}$, triangles: primaries having orientation shifted by $\pm 35^{\circ}$ from solid circles.

different peaks of the $\{110\}$ component with comparable intensities appeared in the primary recrystallized texture, especially on both sides with respect to the cold rolling direction. But the orientations of the secondaries had the same distribution as the left side $\{110\}$ peak of the primaries. When the cold rolling direction was $30^{\circ}$, the peak of the orientation distribution curve of the secondaries is situated closely but is slightly different from that of the primaries of the $\{110\}$ component, to be more exact, between those of the primaries of the $\{110\}$ and $\{111\}$ components.

Figure 4 shows the orientation density of the primaries and the frequency of the secondaries as a function of the rotation angle about TD. The shape of the orientation distribution curve of the secondaries (histogram) was the same as that of the primaries (indicated by circles) with a near- $\{110\}\langle 001\rangle$ orientation in the case of the $0^{\circ}$ cold rolling direction, but it was different by the cold rolling directions of $45^{\circ}$ and $90^{\circ}$. The orientation distribution of the secondaries, however, exhibited the same shape as that of primaries denoted by triangles in Fig. 4 regardless of the cold rolling directions; the triangles show the orientation density of the primaries artificially rotated $\pm 35^{\circ}$ about the [110] axis from a near- $\{111\}\langle 112\rangle$ doublet orientation indicated by solid circles in Fig. 4. The shapes of the orientation distribution curves of the secondaries and the primaries with a near$\{111\}\langle 112\rangle$ orientation about TD or TD' were very sharp regardless of the cold rolling directions.

The orientation density of primaries and the frequency of the secondaries are shown as a function of the rotation angle about RD in Fig. 5. The shape of distribution histogram of the secondaries was the same as the curve of the primaries with $\{h k l\}\langle 112\rangle$ containing the $\{111\}\langle 112\rangle$ orientation, but it was different from $\{h k 0\}\langle 001\rangle$ containing the $\{110\}\langle 001\rangle$ orientation. The orientation distributions of the secondaries and of the primaries with a $\{h k l\}$ $\langle 112\rangle$ orientation about $\mathrm{RD}$ were rather broad regardless of the cold rolling directions.

For the explanation of these experimental results shown in Figs. 3 to 5, it seems necessary to take into consideration the role of not only the $\{110\}$ component but the $\{111\}$ component.

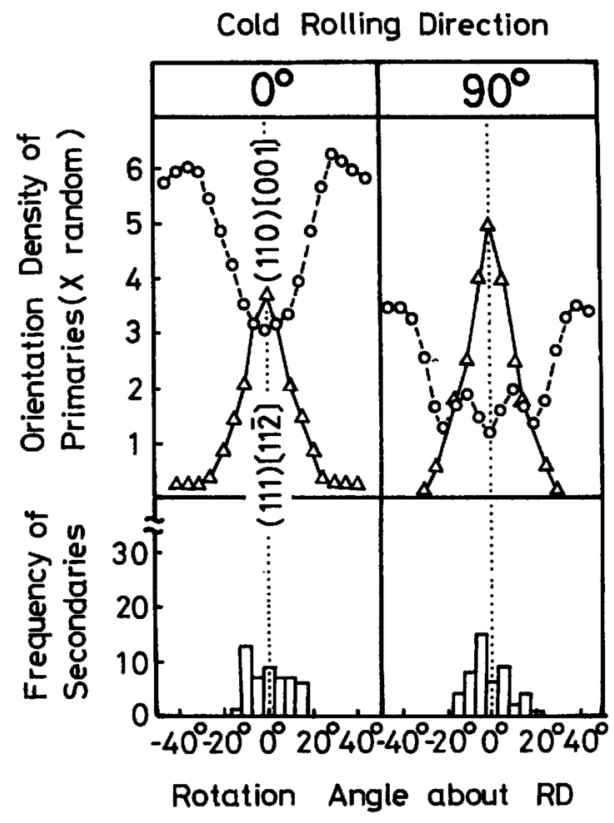

Fig. 5 Comparison between orientation distribution (frequency) of secondaries and orientation density of primaries about RD. Histograms: secondaries, circles: $\{h k 0\}\langle 001\rangle$ primaries, triangles: $\{h k l\}\langle 112\rangle$ primaries. 


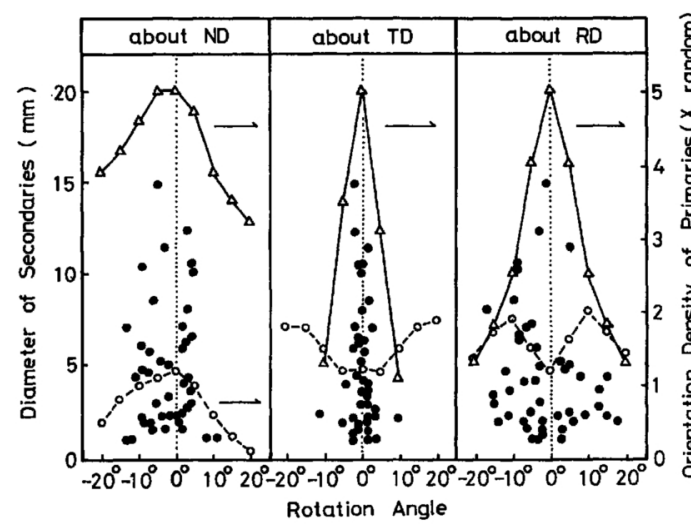

Fig. 6 Comparison between diameter of secondaries and orientation density of primaries about ND, TD and $\mathrm{RD}$ in the case of cold rolling direction of $90^{\circ}$. Solid circles: secondaries, open circles: $\{110\}\langle u v w\rangle$ primaries, triangles: $\{111\}\langle u v w\rangle$ primaries.

Figure 6 shows the orientation dependence of secondary grain size in the case of the $90^{\circ}$ cold rolling direction. Solid circles denote the diameter of secondaries, while the open circles and triangles denote the orientation density of the primaries as replotted from Figs. 3, 4 and 5. It can be seen apparently that the diameter of the secondaries was large when the orientation density of the primaries of the $\{110\}$ or $\{111\}$ component is high.

Figure 7 shows the orientation dependence of the incubation period for the appearance of

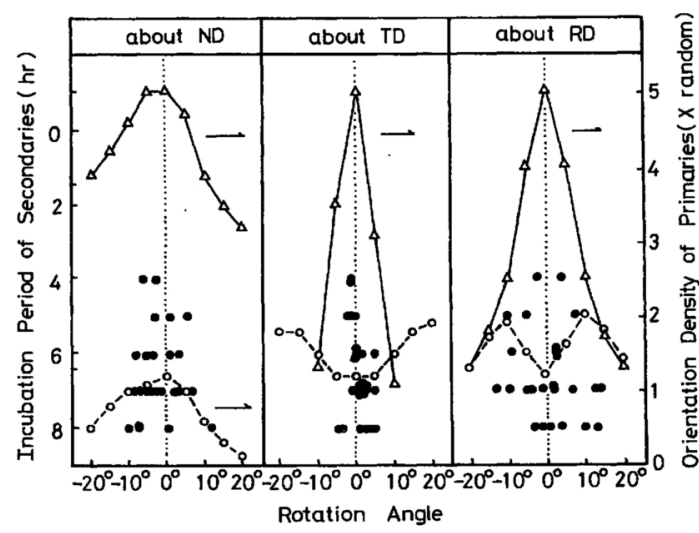

Fig. 7 Comparison between incubation period of secondaries and orientation density of primaries about ND, TD and RD in the case of cold rolling direction of $90^{\circ}$. Solid circles: secondaries, open circles: $\{110\}\langle u v w\rangle$ primaries, triangles: $\{111\}$ $\langle u v w\rangle$ primaries. the secondaries in isothermal annealing at $890^{\circ} \mathrm{C}$ in the case of the $90^{\circ}$ cold rolling direction. The incubation period for the appearance of individual secondary grains was defined as the duration from the beginning of secondary recrystallization annealing to the appearance of individual secondary grains on the specimen surfaces. For this purpose, only one side of the specimens was chemically polished every one hour of annealing, and the appearance and growth of secondaries were observed. Orientation determination of all the secondaries was carried out after the whole specimen underwent complete secondary recrystallization. A tendency can be noticed that the incubation period is short when the high orientation density of the primaries of the $\{110\}$ or $\{111\}$ component is present.

As mentioned above, it can be seen that the parameters describing the behaviour of secondary recrystallization such as the orientation, size and incubation period of secondaries have a good correlation with the orientation distribution of the primaries with a near- $\{110\}\langle 001\rangle$ and $\{111\}<112\rangle$ orientation.

\section{Discussion}

By analogy with some experimental results in single crystals ${ }^{(6) \sim(9)}$ that a primary recrystallized texture with a $\{110\}\langle 001\rangle$ orientation originates from a cold-rolled $\{111\}\langle 112\rangle$ texture, the primary recrystallized matrix with a preferred $\{111\}\langle 112\rangle$ orientation has, in general, been tacitly assumed to be necessary for $\{110\}\langle 001\rangle$ secondary recrystallization. In the present paper, the necessity of coexistence of the primaries with the $\{110\}\langle 001\rangle$ orientation and the $\{111\}\langle 112\rangle$ oriented texture in the primary recrystallized matrix is discussed in terms of asymmetrical primary recrystallized textures analyzed using the special ODF.

It is clear firstly from Figs. $3 \sim 7$ that the secondaries occur in the case of high orientation density of the $\{110\}<001\rangle$ primaries. In other words, the high orientation density of the $\{110\}<001\rangle$ primaries is considered to be necessary for the secondaries of the same orientation to occur. This interpretation agrees with the explanation by Kan et al. ${ }^{(4)}$ As pointed out in 
the explanation of Fig. 3, however, the secondaries in some cases do not always occur in spite of the high orientation density of primaries of the $\{110\}$ component. Therefore, the presence of only one component in the $\{110\}$ primaries cannot be considered the condition necessary for the occurrence of the secondaries, and the following hypothesis is presented.

"The condition necessary for the occurrence of the secondaries is that both $\{110\}$ and $\{111\}$ components of the primaries have a considerably high orientation density."

The results shown in Fig. 3 can be clearly explained by use of this hypothesis. In the case of the $0^{\circ}$ and $60^{\circ}$ cold rolling directions, the orientation distribution curves for the primaries of the $\{111\}$ component are flat and consequently the peaks of distribution histograms of the secondaries are situated at the same orientation as that of the primaries of the $\{110\}$ component. In the case of the $90^{\circ}$ cold rolling direction, the peaks of the orientation distribution curves for the primaries with both $\{110\}$ and $\{111\}$ components are situated at the same point, so that the peak of the secondaries is also situated at the same point as the two peaks in the primaries.

In the case of the $30^{\circ}$ cold rolling direction, the peak of the orientation distribution curve for the primaries of the $\{110\}$ component is not situated at the same point as that of the primaries of the $\{111\}$ component, the peak of the distribution histogram of the secondaries is situated at a middle point between the two peaks of the primaries. In the case of the $45^{\circ}$ cold rolling direction, there are two peaks in the orientation distribution curve for the primaries of the $\{110\}$ component, the peak of the secondaries is situated at the same orientation as one of the two $\{110\}$ peaks, where the density of the primaries of the $\{111\}$ component is higher than the other.

The results shown in Figs. 4 and 5 can be explained in the same manner as mentioned above. Moreover, it can be reasoned that the sharpness of the orientation distribution of the secondaries has a good correlation with that of the primaries of the $\{110\}$ and $\{111\}$ components.

Since the incubation period for the appear- ance of the secondaries is shorter with increasing orientation density of the primaries of the $\{110\}$ or $\{111\}$ component as shown in Fig. 7, the result the increase in size of secondary grains with the orientation density of the primaries of the $\{110\}$ or $\{111\}$ component, Fig. 6, can be reasonably understood. In other words, secondary recrystallized grains with an exact $\{110\}\langle 001\rangle$ orientation have a strong tendency to exhibit a short incubation period and a large grain size.

The orientation relationship between primaries with $\{111\}\langle 112\rangle$ orientation and secondaries with $\{110\}\langle 001\rangle$ orientation during secondary recrystallization could be characterized by the rotating relation of $\pm 35^{\circ}$ about the common $\langle 110\rangle$ axis. The grain boundary with this rotating relation has been suggested to have some coincidence relationship.

Figure 8 shows a relation between the orientation density of the primaries with the $\langle 110\rangle$

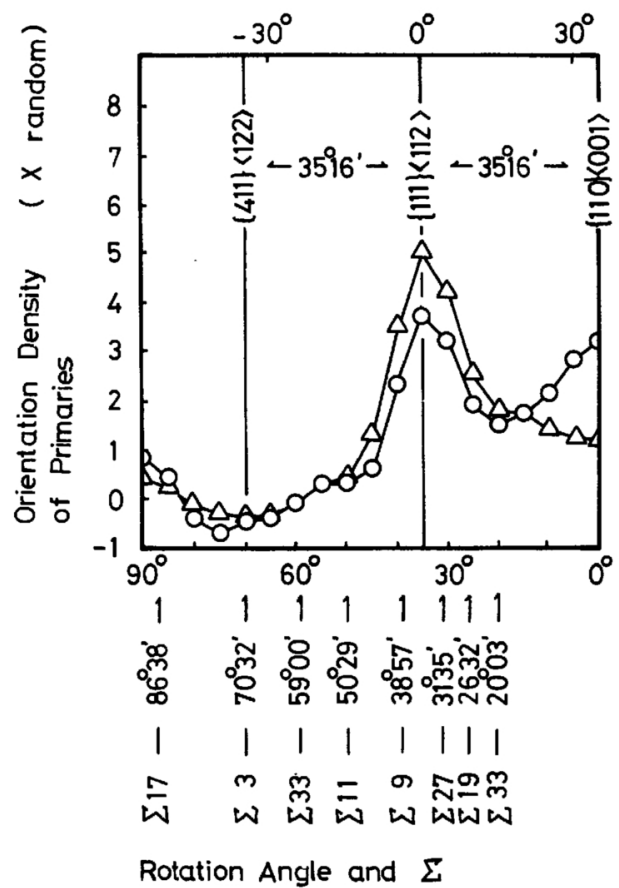

Fig. 8 Relation between orientation density of primaries with $\langle 110\rangle$ axis parallel to TD and rotation angle about $\mathrm{TD}$, referring to distribution of coincidence boundaries about $\langle 110\rangle$ axis. Circles and triangles show the orientation density of primaries in the case of cold rolling directions of $0^{\circ}$ and $90^{\circ}$, respectively. 
axis parallel to TD and the rotation angle about TD, along with the distribution of coincidence boundaries about the $\langle 110\rangle$ axis. Coincidence boundaries with a large $\Sigma$ value $^{(10)}(>\Sigma 33)$ are omitted in this figure. There is no coincidence boundary at $35^{\circ} 16^{\prime}$ about the $\langle 110\rangle$ axis, but five coincidence boundaries are concentrated between $20^{\circ}$ and $50^{\circ}$. A coincidence boundary has its special character within a very narrow region $^{(11)}$ such as $1^{\circ}$ or $2^{\circ}$ in a bi-crystal in which only one grain boundary is present. In the present polycrystalline aggregates, several boundaries enclosing a secondary grain are expected to be in orientations close to some of the five coincidence relations at the same time. This is very probable since the most frequetly observed primary orientations shown in Fig. 8 and therefore boundaries between primary and secondary grains are of orientations distributed mostly in this range. Therefore, a special boundary ${ }^{(12)}$ can account for the most frequently observed boundary misorientation near $35^{\circ}$ as a combined effect of the five coincidence relations concentrated between $20^{\circ}$ and $50^{\circ}$. Graham and $\mathrm{Cahn}^{(13)}$ have obtained some experimental results supporting this consideration. If this interpretation is correct, it is quite reasonable to consider that the behaviour of secondary recrystallization is directly correlated with the orientation density of the primaries having both orientations of near- $\{110\}\langle 001\rangle$ and $\{111\}$ $\langle 112\rangle$.

\section{Summary}

The behaviour of secondary recrystallization in 3\% silicon steel sheets was investigated comparing with the primary recrystallized texture, and the main conclusions were summarized in the following.

(1) The orientation distribution of the secondaries is correlated with the shape of the orientation distribution of the primaries with orientations near $\{110\}\langle 001\rangle$ and $\{111\}\langle 112\rangle$.

(2) As the orientation density of the primaries with the $\{110\}\langle 001\rangle$ or $\{111\}\langle 112\rangle$ orientation becomes higher, the incubation period for the appearance of the secondaries becomes shorter.

(3) As the orientation deviation of the secondaries from the ideal $\{110\}\langle 001\rangle$ orientation becomes smaller, the size of the secondaries becomes larger.

(4) A considerably high orientation density of the primaries with the $\{110\}\langle 001\rangle$ and $\{111\}$ $\langle 112\rangle$ orientation is necessary for the occurrence of the secondaries with the $\{110\}\langle 001\rangle$ orientation.

(5) The above-mentioned phenomena are satisfactorily explained by introducing a hypothesis that in a primary recrystallized texture the $\{110\}\langle 001\rangle$ component becomes a nucleus of secondary recrystallization, and the $\{111\}$ $\langle 112\rangle$ component has some coincidence boundaries, with secondary grains, which are favourable for the growth of the secondary grains.

\section{Acknowledgments}

The authors are deeply indebted to Dr. K. Sanbongi for permitting publication of the present study, and also to Dr. I. Goto and Dr. Y. Inokuchi for a critical reading of the manuscript. The authors also wish to thank Mr. H. Kitagawa for his assistance with computer programs of the three-dimensional crystalline orientation distribution function.

\section{REFERENCES}

(1) R. J. Roe: J. Appl. Phys., 36 (1965), 2024.

(2) H. J. Bunge: Mathematiche Methoden der Texturanalyse, Akademie Verlag, Berlin (1969), p. 15.

(3) J. W. Flowers and Alan J. Heckler: MMM and Inter-Mag. Joint Conference, Pitzburg, (1976).

(4) T. Kan et al.: Synopses of the 1975 Spring Meeting of Japan Inst. Metals, p. 75, (1975) (in Japanese).

(5) T. Abe et al.: J. Iron and Steel Inst. Japan, 55 (1969), S272.

(6) C. G. Dunn: Acta Met., 1 (1953), 163; 2 (1954), 173.

(7) C. G. Dunn and P. K. Koh: Trans. AIME, 206 (1956), 1017.

(8) H. Hu: Acta Met., 8 (1960), 124; Trans. Met. Soc. AIME, 221 (1961), 130.

(9) O. Hashimoto, N. Ohashi and K. Fujimoto: J. Iron and Steel Inst. Japan, 57 (1971), 1167.

(10) D. G. Brandon, B. Ralph, S. Ranganathan and M. S. Wald: Acta Met., 12 (1964), 813.

(11) Y. Ishida: Bull. Japan Inst. Metals, 9 (1970), 156.

(12) K. T. Aust and J. W. Rutter: Trans. Met. Soc. AIME, 215 (1959), 820.

(13) C. D. Graham, Jr. and R. W. Cahn: Trans. AIME, 206 (1956), 517; Journal of Metals (May 1956). 